\title{
Case report: methrotexate induced neurotoxicity mimicking stroke, following leukoencephalopathy
}

\begin{abstract}
Acute lymphocytic leukemia (ALL) is the most prevalent cancer in children. The definitive diagnosis is made specially through myelogram and bone marrow biopsy. Methotrexate (MTX) is an essential drug in the treatment of childhood acute lymphoblastic leukemia (ALL) and may be used alone or in combination with other drugs in a multi-chemotherapy approach. However, MTX has some several side effects attributed to it, such as neurotoxicity, especially administrated intrathecal. Neurotoxicity can be acute, subacute or long-term encephalopathy. The case presented is classical evolution of a transient encephalopathy with a stroke-like manifestation. It is a rare early adverse effect of MTX medication and resolves in a short period of time with medication for vasodilation (aminophylline). However, focal white matter lesions are firstly shown in diffusion weighted imaging (DWI) and within days can be seen in other sequences. Abnormal diffusion areas vanishes as the improvement of clinical symptoms.
\end{abstract}

Keywords: acute, leukemia, children, methotrexate, neurotoxicity, encephalopathy
Volume 3 Issue 3 - 2017

Lederman HM,' Grassi DC,' Camargo MV,2 Sousa AVL, ${ }^{3}$ Carneiro SGB, ' Tostes VS, ${ }^{3}$ Silva $F A B^{\prime}$

'Department of Diagnostic Imaging, Federal University of Sao Paulo, Brazil

${ }^{2}$ Diagnostic Center at Pediatric Oncology Institute, Brazil

${ }^{3}$ Hematology at Pediatric Oncology Institute, Brazil

Correspondence: Henrique Manoel Lederman, Department of Diagnostic Imaging, Federal University of Sao Paulo and IOP -GRAACC, R. Botucatu, 743 - Vila Clementino, São Paulo-SP, Brazil,Tel +55 II 50808586, Email lederman@graacc.org.br

Received: June 16, 2017 | Published: July 07, 2017
Abbreviations: ALL, acute lymphocytic leukemia; MTX, methotrexate; DWI, diffusion weighted imaging; CT, computerized tomography; MRI, magnetic resonance imaging; ADC, apparent diffusion coefficient

\section{Introduction}

Acute lymphocytic leukemia (ALL) is the most prevalent cancer in children. It has various presentations secondary to a deregulated proliferation of the lymphocytic lineage and consequent accumulation of immature lymphocytes in healthy tissues, leading to deregulation of its normal function. ${ }^{1}$ The diagnostic suspicion is made through the signs and symptoms that are secondary to the accumulation of blasts in the bone marrow, generating insufficiency of the bone marrow and later invasion of the tissues. However, the definitive diagnosis is made through myelogram, bone marrow biopsy and imunophenotyping/ cytogenetics. $^{2}$

Methotrexate (MTX) is an essential drug in the treatment of childhood acute lymphoblastic leukemia (ALL) and may be used alone or in combination with other drugs in a multi-chemotherapy approach. It can be administrated intravenously as well as intrathecally, being crucial for prophylaxis and treatment of sanctuary sites, including the CNS. However, it demonstrates several toxic effects such as mucositis, myelosuppression, nephrotoxicity, hepatotoxicity and neurotoxicity.,

Special attention is given to neurotoxicity, and may cause acute, subacute, and long-term encephalopathy. Clinically, manifestations can be stroke-like symptoms, encephalopathy, seizures, and/or aphasia, and are usually reversible, but occasionally causes patient death or severe sequelae, for this reason is important to monitor patients in risk. Magnetic resonance imaging is very useful in diagnosis, showing alterations in diffusion-weighted imaging of a precocious way. ${ }^{5,6}$

\section{Case presentation}

S.O.M, a 16-year-old male patient, was referred from an outside institution to the Pediatric Oncology Institute - GRAACC with complaint of ankle pain joint swelling in both knees, making it impossible to walk. He also reported weight loss, prostration and fever. During the diagnostic investigation myelogram was performed, leading to the diagnosis of acute lymphoblastic leukemia subtype B. After that, chemotherapy with MTX, doxorubicin and cyclophosphamide was started. After 9 days of receiving intrathecal MTX, the patient reported headache associated with decreased strength in right arm and aphasia.

A CT (Computerized Tomography) scan of the skull was requested and did not show any signs of intracranial hemorrhage. Subsequently, magnetic resonance imaging (MRI) of the brain was performed, evidencing two focal areas of high signal in DWI in bilateral centrum semiovale (Figures 1-5). Considering that the treatment wasn't finished and still consisted in high and continuous doses of MTX, aminophylline was introduced in order to prevent/reverse the effects of the subacute ischemic event due to the medication.

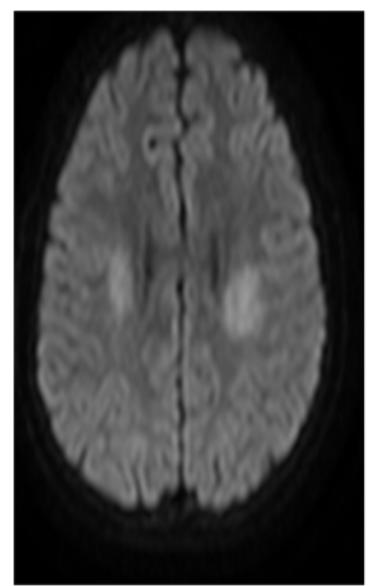

Figure I Focal and symmetrical regions of restricted diffusion on DWI on centrum semiovale. 


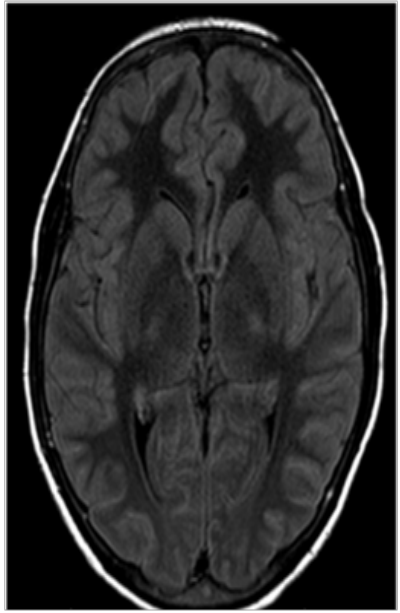

Figure 2 FLAIR images showing no abnormalities.

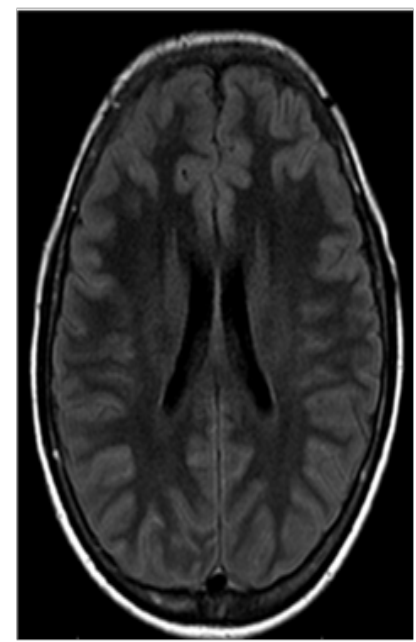

Figure 3 FLAIR images showing no abnormalities.

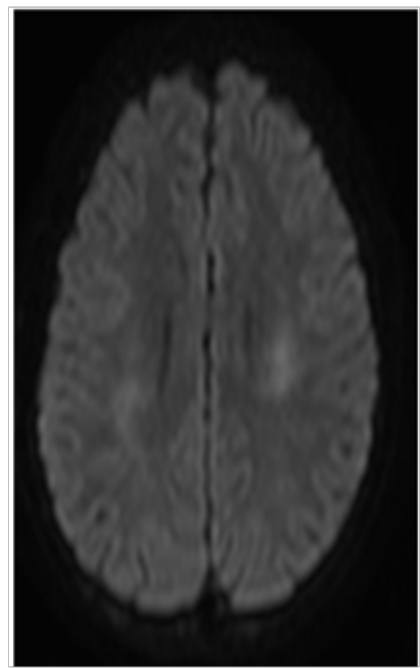

Figure 4 Focal and symmetrical regions of restricted diffusion on DWI on centrum semiovale.

Two days after aminophylline was started, there was improvement of areas with high DWI signal. Four days later, the patient recovered movements on the face and upper limbs. In the two- week follow-up, demonstrated areas of high signal in T2 in correspondence previous areas of change of signal in DWI. (Figures 6-8).

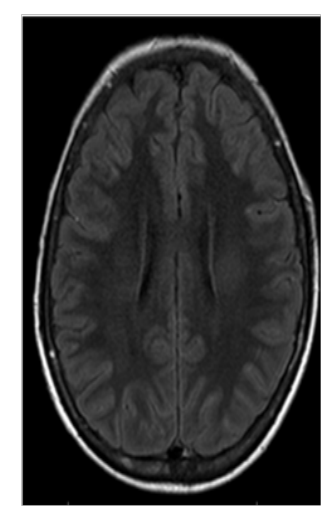

Figure 5 with no correspondence in FLAIR images.

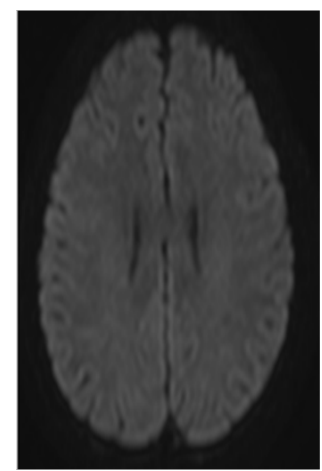

Figures $6(\mathrm{DWl})$ No more identification of the focal areas of restricted diffusion in both centrum semiovale.

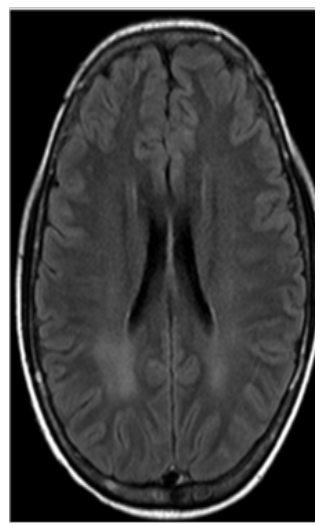

Figure 7 (FLAIR) now it is possible to identify correspondently high signal FLAIR images.

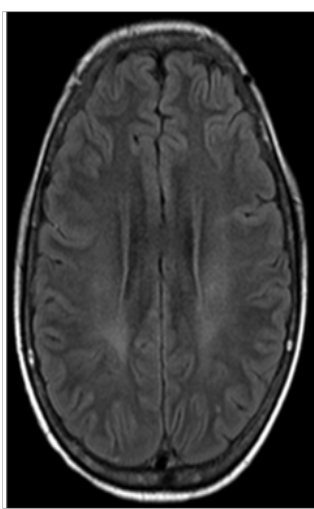

Figure 8 (FLAIR) now it is possible to identify correspondently high signal FLAIR images. 


\section{Discussion}

Although multiple drugs are used in addition to MTX, the acute neurotoxicity reported in patients with ALL undergoing therapy is usually attributed to MTX. ${ }^{7}$ The pathogenesis of encephalopathy is not well understood nowadays and is postulated to be related to multiple dysregulated neuronal biochemical processes in addition to a potential direct neurotoxic effect of MTX. Relevant processes are inhibition of the enzyme dihydrofolate reductase, preventing cell replication; disturbances of myelin and glucose metabolism. In addition, causes a relative excess of homocysteine, which is thought to induce smallvessel vasculopathy. ${ }^{7-9}$

Both high-dose intravenous MTX and intrathecal MTX are associated with parenchymal damage and the mechanism of the changes may represent metabolic effects, increased capillary permeability, transient vasospasm, small vessel occlusion, or the demyelinating process itself. ${ }^{10}$ MTX neurotoxicity could be acute (within hours), subacute (days to weeks after administration), and chronic (after months andyears) encephalopathy. Clinically, acute and subacute neurotoxicity usually results in stroke like symptoms, such as aphasia, weakness, sensory deficits, ataxia, and seizures. ${ }^{11}$

The imaging modality of choice is MRI, because it has a high sensitivity and specificity for relevant differential diagnosis such as cerebral metastases, opportunistic infection, hemorrhage, dural venous obstruction or ischemic infarction. In acute/subacute stroke like MTX-induced neurotoxicity, DW and T2 hyperintensities are typically located in the periventricular white matter, particularly in the centrum semiovale, and often seen only on DWI (diffusion weighted images). ${ }^{12}$

The MR imaging findings consists of increased signal intensity on DWI with correlated hypointensity on the ADC map are indicative diminished random water motion, usually associated with irreversible cytotoxic injury. However, it can be identified in acute MTX neurotoxicity related to cytotoxic edema and is not necessarily associated with irreversible cell death as the proposed mechanism. After recovery, DWI findings are usually normal, but T2 and/or FLAIR (Fluid-attenuated inversion recovery) may show residual abnormalities until 3weeks. Reversibility of markedly decreased in apparent diffusion coeficient (ADC) values without a clinically apparent permanent deficit is in striking contrast with findings in acute ischemia. ${ }^{13}$

\section{Conclusion}

The case here presented showed the expected evolution of a subacute transient encephalopathy with a stroke-like manifestation. It is common adverse effect of MTX medication and it tends to resolve spontaneously within few days. However, once it is diagnosed, aminophylline plays a special role on reversing and preventing from further MTX- toxicity related symptoms. MRI is fundamental on the diagnosis, showing focal white matter lesions which are firstly seen in
DWI and within days, these areas vanishes in DWI and appears in T2 images. As these focal areas disappear in DWI, there is a correlation with clinical symptoms improvement.

\section{Acknowledgements}

None.

\section{Conflict of interest}

Author declares that there is no conflict of interest.

\section{References}

1. Mitchell C, Hall G, Clarke RT. Acute leukaemia in children: diagnosis and management. BMJ. 2009;338:b2285.

2. William L Carroll, Deepa Bhojwani, Dong-Joon Min, et al. Pediatric Acute Lymphoblastic Leukemia Hematology. Research profiles. 2003:1-30.

3. Bhojwani D, Sabin ND, Pei D, et al. Methotrexate-Induced Neurotoxicity and Leukoencephalopathy in Childhood Acute Lymphoblastic Leukemia. J Clin Oncol. 2014;32(9):949-959.

4. Ruggiero A, Conter V, Milani M, et al. Intrathecal chemotherapy with antineoplastic agents in children. Paediatr Drugs. 2001;3(4):237-246.

5. Carroll WL, Bhojwani D, Min DJ. Pediatric acute lymphoblastic leukemia. Drugs \& Diseases. 2003. p. 102-131.

6. Agarwal A, Vijay K, Thamburaj K, et al. Transient leukoencephalopathy after intrathecal methotrexate mimicking stroke. Emerg Radiol. 2011;18(4):345-347.

7. Rollins N, Winick N, Bash R, et al. Acute Methotrexate Neurotoxicity: Findings on Diffusion-Weighted Imaging and Correlation with Clinical Outcome. AJNR Am J Neuroradiol. 2004;25(10):1688-1695.

8. Kishi S, Greiner J, Cheng C, et al. Homocysteine, pharmacokinetics, and neurotoxicity in children with leukemia. J Clin Oncol. 2003;15(16):3084-3091.

9. Brugnoletti F, Morris EB, Laningham FH, et al. Recurrent intrathecal methotrexate induced neurotoxicity in an adolescent with acute lymphoblastic leukemia: Serial clinical and radiologic findings. Pediatr Blood Cancer. 2009;52(2):293-295.

10. Agarwal A, Vijay K, Thamburaj K, et al. Transient leukoencephalopathy after intrathecal methotrexate mimicking stroke. Emerg Radiol. 2011;18(4):345-347.

11. Sandoval C, Kutscher M, Jayabose S, et al. Neurotoxicity of Intrathecal Methotrexate: MR Imaging Findings. American Journal of Neuroradiology. 2003;24(9):1887-1890.

12. Eichler AF, Batchelor TT, Henson JW. Diffusion and perfusion imaging in subacute neurotoxicity Diffusion and perfusion imaging in subacute neurotoxicity following high-dose intravenous methotrexate. Neuro Oncol. 2007;9(3):373-377.

13. Küker W, Bader P, Herrlinger U, et al. Transient encephalopathy after intrathekal methotrexate chemotherapy: diffusion-weighted MRI. J Neurooncol. 2005;73(1):47-49. 